\title{
Factores asociados a las prácticas de enseñanza docentes con apoyo de las tecnologías de la información y comunicación
}

\author{
Claudia Gabriela Arreola Olivarría \\ María Teresa Fernández Nistal \\ Javier José Vales García \\ Instituto Tecnológico de Sonora. México. \\ gaarreoli@gmail.com \\ mfernandez@itson.edu.mx \\ javiervales@yahoo.com.mx
}

Pedro Antonio Sánchez Escobedo

Universidad Autónoma de Yucatán. México.

psanchez@correo.uady.mx

Recibido: $13 / 2 / 2021$

Aceptado: 22/7/2021

Publicado: 31/1/2022

\section{Resumen}

La integración de las tecnologías de la información y comunicación (TIC) por parte de los docentes en el proceso de enseñanza es importante para mejorar la calidad de la educación. El presente estudio se propuso examinar las relaciones de la habilidad digital, la usabilidad, la utilidad y la gestión directiva con la frecuencia con que maestros de educación primaria utilizaban TIC en sus prácticas de enseñanza. Participaron en el estudio 252 docentes $(M$ edad $=36,5$ años, $D E=10,1)$ del Estado de Sonora (México). Se realizó un estudio transversal de alcance relacional. Se utilizaron escalas de autorreporte para medir las variables involucradas en el estudio. Se calculó un modelo de regresión lineal múltiple. Se encontró que tanto variables relativas al docente (habilidad digital y usabilidad) como al director (gestión directiva) se asociaban positivamente con las prácticas de enseñanza con apoyo en tecnología. Se concluyó que la integración de las TIC en la enseñanza por parte de los docentes se relacionaba con variables del docente y de los directores.

Palabras clave: docentes; usabilidad; utilidad; habilidad digital; gestión directiva

Resum. Factors associats a les pràctiques d'ensenyament docents amb suport de les tecnologies de la informació $i$ la comunicació

La integració de les tecnologies de la informació i la comunicació (TIC) per part dels docents en el procés d'ensenyament és important per millorar la qualitat de l'educació. Aquest estudi es va proposar d'examinar les relacions de l'habilitat digital, la usabilitat, la utilitat i la gestió directiva amb la freqüència amb què docents d'educació primària utilitzen TIC a les pràctiques d'ensenyament. Van participar a l'estudi 252 docents $(M$ edat $=36,5$ anys, $D E=10,1$ ) de l'estat de Sonora, Mèxic. Es va fer un estudi transversal d'abast relacional. Es van utilitzar escales d'autoreport per mesurar les variables involucrades a l'estudi. Es va calcular un model de regressió lineal múltiple. Es va trobar que tant les 
variables relatives al docent (habilitat digital i usabilitat) com al director (gestió directiva) s'associen positivament amb les pràctiques d'ensenyament amb suport de tecnologia. Es va concloure que la integració de les TIC a l'ensenyament per part dels docents es relaciona amb variables del docent i dels directors.

Paraules clau: docents; usabilitat; utilitat; habilitat digital; gestió directiva

\title{
Abstract. Factors associated with teaching practices with the support of information and communication technologies
}

The integration of information and communication technologies (ICT) by teachers in the teaching process is essential for improving the quality of education. The present study examines the relationships of digital ability, usability, utility, and management with the frequency with which elementary school teachers use ICT in their teaching practices. The study sample comprises 252 teachers $(\mathrm{M}$ age $=36.5$ years, $\mathrm{SD}=10.1)$ of Sonora, Mexico. A cross-sectional relational design was used. Self-report scales were used to measure the variables. A multiple linear regression model has shown that variables related to both the teacher (digital ability and usability) and the principal (management) are positively associated with technology-supported teaching practices. We conclude that the integration of ICT in teaching by teachers is related to variables regarding the teacher and principals.

Keywords: teachers; usability; utility; digital skills; management

\author{
Sumario \\ 1. Introducción 4. Conclusiones \\ 2. Método Referencias bibliográficas
}

3. Resultados

\section{Introducción}

Las tecnologías de la información y comunicación (TIC) modifican y facilitan el desarrollo de las sociedades actuales. Las TIC cambian la forma en que los estudiantes se comunican, adquieren información y desarrollan habilidades intelectuales (Burns y Gottschalk, 2019; Organisation for Economic Cooperation and Development, 2017). Diversos estudios sugieren que las TIC favorecen la autorregulación y el desempeño académico de los estudiantes (Hu et al., 2018; Skryabin et al., 2015). Además, algunos autores afirman que la capacidad de las TIC para reducir obstáculos, tales como el tiempo y la distancia entre los aprendices y los maestros, facilita el aprendizaje colaborativo (Hernández-Martín y Martín de Arriba, 2017; Xiao, Lin y Hu, 2019).

En general, la evidencia empírica muestra que las TIC contribuyen de forma significativa a la mejora del proceso de enseñanza-aprendizaje (Comi et al., 2017; Odu y Mireku, 2016). Sin embargo, en México los docentes de educación básica utilizan con poca frecuencia las TIC en sus prácticas de enseñanza (Gil-Flores, Rodríguez-Santero y Torres-Gordillo, 2017; Gómez-Domínguez 
et al., 2019). Por lo tanto, examinar los elementos que limitan el uso de las TIC por parte del profesorado en el desarrollo de su profesión es importante para mejorar la calidad de la educación en el nivel básico. Si bien son complejos los factores asociados con el uso de las TIC por parte de los maestros (GilFlores et al., 2017; Lawrence y Tar, 2018), diversos autores sugieren qué aspectos relativos al docente y al director resultan esenciales para predecir la integración de la tecnología en la enseñanza (Ifinedo, Rikala y Hämäläinen, 2020; Muli et al., 2017).

\subsection{Variables del docente}

La integración de las TIC en la enseñanza involucra el uso de las tecnologías por parte de los docentes para facilitar el aprendizaje de sus estudiantes. Diversas investigaciones sugieren que esta integración se relaciona con las habilidades tecnológicas, la percepción de usabilidad y la utilidad percibida por parte de los docentes de las TIC como recursos de apoyo a la enseñanza (Ifinedo et al., 2020; Tarhini et al., 2016).

El modelo de conocimiento tecnológico, pedagógico y de contenido - el TPACK (Mishra y Koehler, 2006) — sostiene que la adopción de las TIC por parte de los docentes se relaciona con la alfabetización tecnológica de los mismos, particularmente con las habilidades para utilizar las tecnologías como apoyo a la enseñanza. La evidencia empírica consistente con lo propuesto en el modelo TPACK constata qué habilidades digitales de los docentes se relacionan con la utilización de las tecnologías en la enseñanza (Glasserman-Morales y Manzano-Torres, 2016; Ifinedo et al., 2020). Estas habilidades tecnológicas comprenden el dominio de aspectos técnicos y pedagógicos. Los aspectos técnicos se refieren a las destrezas para dominar el funcionamiento de las TIC (Almerich et al., 2016; Ríos-Ariza, Gómez-Barajas y Rojas-Polanco, 2018). Por su parte, la habilidad pedagógica implica el uso de los recursos digitales a favor del aprendizaje de los estudiantes, lo que involucra generar estrategias para utilizar estas herramientas como apoyo a su enseñanza (Chai et al., 2017; Kiray, Celik y Colakoglu, 2018).

Por otra parte, dentro del modelo de aceptación tecnológica —el TAM (Davis, 1989)—, la percepción de usabilidad y de utilidad de las TIC son factores relacionados con el uso de los recursos tecnológicos en la enseñanza. En el contexto escolar, la usabilidad se refiere a la percepción del docente acerca de la facilidad con que puede utilizar las TIC como apoyo a la enseñanza en los salones de clases (Beltrán-Sánchez, García-López y Ramírez-Montoya, 2015; Sánchez-Prieto, Olmos-Migueláñez y García-Peñalvo, 2015). Por su parte, la utilidad refleja la percepción del maestro acerca de los beneficios que le aporta el uso de las TIC en su desempeño (Tarhini et al., 2016). Tanto la usabilidad (Bai, Wang y Chai, 2021; Cataldo, 2015) como la utilidad percibida de las TIC se relacionan de forma positiva con la integración de las tecnologías por parte de los docentes en la enseñanza (Sherer, Siddiq y Teo, 2015; Siddiq, Sherer y Tondeur, 2016). 


\subsection{Variables del director}

Un grupo considerable de estudios sugiere que el director influye en la eficacia escolar (Mendoza-Orellana, 2019; Stein, Macaluso y Stanulis, 2016). Diversos autores consideran que la gestión directiva influye en la innovación de las prácticas de enseñanza por parte de los docentes (Daly, Liou y Moolenaar, 2014). Según la Organización de las Naciones Unidas para la Educación, la Ciencia y la Cultura (2014), la gestión directiva involucra actividades del director encaminadas a conducir de forma eficiente las prácticas docentes y las tareas educativas con el fin de obtener mejores resultados de aprendizaje en los estudiantes. La gestión directiva eficiente se relaciona con la percepción de apoyo por parte de los docentes para desarrollar sus prácticas de enseñanza (Ilknur y Yeliz, 2018; Kars e Inandi, 2018).

Pese a la evidencia existente respecto al hecho de que la gestión de los directores influye en las prácticas docentes (Lim, Gronlund y Andersson, 2015; Stein et al., 2016), las investigaciones sobre la influencia de esta variable en la integración de las TIC son escasas en México. Los limitados estudios sobre el tema coinciden en señalar que la gestión directiva influye en la integración de las TIC en la enseñanza. Al respecto, se reporta que los directores que poseen una percepción positiva de las TIC y que consideran importante la disponibilidad de los recursos informáticos fomentan el uso de las tecnologías por parte de los docentes (Wu et al., 2019). En otros estudios se constata que tanto las actitudes positivas como el liderazgo transformacional, instruccional y tecnológico de los directores contribuyen al éxito de utilización de las TIC en la escuela (Navaridas-Nalda, Emeterio, Fernández-Ortiz y Arias-Oliva, 2020; Machado y Chung, 2015). Finalmente, Arokiasamy, Abdullah e Ismail (2015) muestran que el liderazgo transformacional de los directores se relaciona positivamente con el uso de las computadoras en la escuela.

\subsection{Objetivo}

En este contexto, el presente análisis pretende contribuir a facilitar la comprensión de los factores vinculados con el uso de las TIC por parte de maestros de educación primaria de Sonora (México) en sus prácticas de enseñanza. Para esto se propuso examinar las relaciones entre variables del docente (habilidad digital, usabilidad y utilidad) y del director (gestión directiva) con la integración de las TIC en la labor educativa de los docentes.

\subsection{Hipótesis}

H1. La habilidad digital, la usabilidad, la utilidad y la gestión directiva se relacionan positivamente con la integración de las TIC en las prácticas de enseñanza por parte de docentes de educación primaria. 


\section{Método}

\subsection{Tipo de estudio}

Se realizó una investigación no experimental, lo que significa que no se manipularon las variables. El diseño fue transeccional, ya que se midieron las variables en un momento determinado de tiempo. El estudio se propuso llegar a un alcance relacional explicativo, al examinar las relaciones entre diversas variables (Johnson y Christensen, 2017).

\subsection{Participantes}

La muestra de los docentes fue extraída de 63 escuelas primarias públicas de una ciudad de Sonora (México) $(n=504)$. De forma no probabilística se seleccionaron cuatro maestros de cada escuela, 126 de primaria baja (de primero a tercer grado) y 126 de primaria alta (de cuarto a sexto grado). En total participaron en el estudio 252 docentes, con edades entre 21 y 62 años $(M$ edad $=36,5$ años, $D E=10,1$ ). Similar a otras escuelas primarias públicas de México, los centros involucrados en el estudio atendían a estudiantes de diversas clases sociales, especialmente de clase baja y media (Instituto Nacional de Estadística y Geografía, 2018).

Un poco más de la mitad de los docentes de la muestra (el 52\%) no reportaron capacitación con respecto al uso pedagógico de las TIC en los últimos tres años, mientras que el $25 \%$ menciona haber tomado únicamente un curso en el mismo periodo de tiempo. El 92\% cuenta con servicio de internet en el hogar; casi todos disponen de celular inteligente con acceso a la red (el 97\%); el $92 \%$ posee un portátil personal, y el 38\%, una computadora de escritorio en su casa.

\subsection{Instrumentos}

Se utilizaron cinco instrumentos de autorreporte para medir las variables involucradas en el estudio. A continuación se menciona la variable y se describe el instrumento utilizado para su medición.

Prácticas de enseñanza con apoyo de las TIC. Se elaboró exprofeso para el estudio a partir de otros instrumentos reportados en la literatura (Bas, Kubiatko y Sünbül, 2016; Comi et al., 2017). Se elaboraron 15 ítems, los mismos que se enviaron a evaluación por el juicio de expertos ( 5 investigadores en la temática y 2 docentes de educación primaria). Los expertos valoraron la relevancia de cada ítem. La evaluación se realizó con una escala tipo Likert de cuatro puntos (por ejemplo: «El ítem es relevante para medir el constructo»: 1 = nada relevante, 2 = no posee relevancia a no ser que se realicen correcciones mayores, $3=$ relevante y $4=$ muy relevante). Se seleccionaron para ser incluidos en la escala 6 ítems con un índice de validez de contenido (IVC) mayor a 0,70 (Almanasreh, Moles y Chen, 2019). Los 6 ítems miden el apoyo a la enseñanza (3 ítems, por ejemplo: «Uso juegos o simulaciones apoyados con tecnología 
en mi salón de clases»; $\alpha=0,72)$ y la comunicación con estudiantes y otros (3 ítems, por ejemplo: «Me apoyo en la tecnología para comunicar a los padres de familia información acerca de la escuela y sus hijos»; $\alpha=0,78)$. El formato de respuesta fue tipo Likert con cinco opciones, desde 0 (nunca) hasta 4 (siempre). Los resultados del análisis factorial confirmatorio (AFC) sugieren que el modelo de medición se ajusta a los datos $\left(X^{2}=8,89, g l=8, p=0,352\right.$; SRMR $=$ 0,$04 ; \mathrm{CFI}=0,98 ; \mathrm{TLI}=0,97$; RMSEA = 0,02, IC $90[0,01,0,08]$ ).

Habilidad digital docente. Se midió con la escala de competencias tecnológicas (Maddox, 2004) conformada por 6 ítems (por ejemplo: «Utilizar dispositivos como escáneres, cámaras digitales y/o cámara de video con computadora y software para apoyar mi enseñanza»; $\alpha=0,89$ ). Se utilizó el método de retrotraducción para traducir el instrumento del idioma inglés al español. El formato de respuesta fue tipo Likert con cinco opciones, desde 0 (nada hábil) hasta 4 (muy hábil). El AFC verificó el ajuste del modelo a los datos $\left(X^{2}=\right.$ $16,99, g l=9, p=0,049 ;$ SRMR = 0,034; CFI = 0,99; TLI = 0,98; RMSEA = 0,06, IC $90[0,01,0,08])$.

Usabilidad. La escala se desarrolló exprofeso para el estudio con el fin de medir la percepción del docente sobre la facilidad de uso de las TIC como apoyo a la enseñanza. Se elaboraron 8 ítems, los mismos que fueron evaluados por expertos (3 investigadores en la temática y 5 docentes de educación primaria). Los expertos valoraron la relevancia de cada ítem. La evaluación se realizó con una escala tipo Likert de cuatro puntos (por ejemplo: «El ítem es relevante para medir el constructo"; $1=$ nada relevante, $2=$ no posee relevancia a no ser que se realicen correcciones mayores, $3=$ relevante y $4=$ muy relevante). Se seleccionaron para ser incluidos en la escala 5 ítems (por ejemplo: «Es fácil utilizar el software o plataformas educativas para apoyar la enseñanza»; $\alpha=0,82$ ) con IVC mayor a 0,70 (Almanasreh et al., 2019). Se utilizó un formato tipo Likert con cuatro opciones de respuesta, desde 0 (totalmente en desacuerdo) hasta 3 (totalmente de acuerdo). El AFC muestra índices adecuados de ajuste del modelo $\left(X^{2}=7,23, g l=4, p=0,124 ; \mathrm{SRMR}=0,010\right.$; CFI $=0,99 ; \mathrm{TLI}=$ $0,98$; RMSEA $=0,062$, IC $90[0,01,0,08])$.

Utilidad. La escala se diseñó exprofeso para el estudio con el fin de medir la percepción de los docentes acerca de la utilidad de las TIC como apoyo a la enseñanza. Se elaboraron 10 ítems que fueron sometidos al juicio de expertos (3 investigadores en la temática y 3 docentes de educación primaria). Los expertos evaluaron la relevancia de cada ítem. La evaluación se realizó con una escala tipo Likert de cuatro puntos (por ejemplo: «El ítem es relevante para medir el constructo"; 1 = nada relevante, 2 = no posee relevancia a no ser que se realicen correcciones mayores, $3=$ relevante y $4=$ muy relevante). Se seleccionaron para ser incluidos en la escala 6 ítems (por ejemplo: «Apoyan la autorregulación del aprendizaje»; $\alpha=0,90$ ) con IVC mayor a 0,70 (Almanasreh et al., 2019). El formato de respuesta fue de tipo Likert con cuatro opciones, desde 0 (totalmente en desacuerdo) hasta 3 (totalmente de acuerdo). El AFC muestra índices adecuados de ajuste del modelo $\left(X^{2}=18,64, g l=9, p=0,028\right.$; SRMR $=0,012 ; \mathrm{CFI}=0,99 ; \mathrm{TLI}=0,98 ; \mathrm{RMSEA}=0,071, \mathrm{IC} 90[0,022,0,008])$. 
Gestión directiva. Se elaboró exprofeso para el estudio con base en las funciones de la gestión directa establecidas por la Secretaría de Educación Pública (2017). Mide las actividades del director para fomentar el uso de las TIC en los docentes (por ejemplo: «Realiza diagnósticos de la disponibilidad y funcionalidad de los recursos tecnológicos de la escuela»; $\alpha=0,91$ ). Se elaboraron 14 ítems, los mismos que se enviaron a evaluación por parte de expertos ( 3 investigadores en la temática y 5 directores de educación primaria), que valoraron la relevancia de cada ítem. La evaluación se realizó con una escala tipo Likert de cuatro puntos (por ejemplo: «El ítem es relevante para medir el constructo"; 1 = nada relevante, 2 = no posee relevancia a no ser que se realicen correcciones mayores, $3=$ relevante y $4=$ muy relevante). Se seleccionaron para ser incluidos en la escala 7 ítems con IVC mayor a 0,70 (Almanasreh et al., 2019). Se contestó en formato tipo Likert con opciones de respuesta, desde 0 (nunca) hasta 4 (siempre). El AFC muestra índices de ajuste adecuados del modelo $\left(X^{2}=25,93, g l=13, p=0,017\right.$; SRMR $=0,041$; CFI $=0,98 ;$ TLI $=$ 0,98 ; RMSEA = 0,06, IC $90[0,03,0,08]$ ).

\subsection{Procedimiento}

Se solicitó formalmente el consentimiento de los directivos de los centros escolares. Posteriormente se contactó con los docentes para explicarles el propósito del estudio y pedirles su participación voluntaria en el mismo. Se aseguró la confidencialidad de la información brindada por los docentes. Los instrumentos fueron administrados en las escuelas por los investigadores participantes en la investigación.

\subsection{Análisis estadísticos}

Los datos perdidos en todas las variables fueron menores al 5\% y se trataron por el método de imputación múltiple disponible en el software SPSS 25. Se realizaron análisis factoriales confirmatorios de los modelos de medición para cada una de las variables con apoyo de AMOS. El cálculo de los modelos se llevó a cabo con el método de máxima verosimilitud y un Boostrap asociado (500 repeticiones, $95 \%$ de intervalo de confianza). El método de Boostrap es utilizado para evitar que las estimaciones sean afectadas por problemas de normalidad multivariada (Byrne, 2016). Posteriormente se realizaron análisis descriptivos y correlacionales de las variables involucradas en el estudio. Por último, se calculó un modelo de regresión lineal jerárquica con apoyo del SPSS 25 para analizar las relaciones entre las variables independientes (habilidad digital, usabilidad, utilidad y gestión directiva) y la dependiente (prácticas docentes con apoyo de las TIC). En el primer modelo se incluyeron las variables relativas al docente (habilidad digital, usabilidad y utilidad) como variables de entrada, y en el segundo modelo, las referidas al director (gestión directiva). 


\section{Resultados}

Como parte del análisis preliminar se calcularon las medias, las desviaciones estándar y las correlaciones entre las variables involucradas en el estudio. Se encontró que los docentes reportaron utilizar las TIC en sus prácticas de enseñanza ocasionalmente, manifestaron acuerdo acerca de su utilidad, se percibieron como medianamente hábiles para utilizar las tecnologías en la enseñan$\mathrm{za}$, se mostraron de acuerdo en cuanto a la usabilidad de las TIC y percibieron que únicamente de forma ocasional los directores promovían que utilizaran las TIC en el desempeño de su profesión. Además, se observaron correlaciones positivas entre las habilidades, la utilidad, la facilidad de uso, la gestión directiva y las prácticas de enseñanza con apoyo de las TIC. Esto implica que estas variables favorecen la utilización de las TIC en la enseñanza por parte de los docentes. Finalmente, la gestión directiva no se correlacionó significativamente con utilidad ni con usabilidad.

Tabla 1. Media, desviación estándar y correlación entre las variables de estudio

\begin{tabular}{lccccccc}
\hline Variable & $\boldsymbol{M}$ & $\boldsymbol{D E}$ & $\mathbf{1}$ & $\mathbf{2}$ & $\mathbf{3}$ & $\mathbf{4}$ & $\mathbf{5}$ \\
\hline 1. Prácticas de enseñanza & 2,11 & 0,70 & - & & & & \\
2. Utilidad & 2,24 & 0,55 & $0,24^{* *}$ & - & & & \\
\hline 3. Habilidad digital & 2,62 & 0,92 & $0,47^{* *}$ & $0,17^{*}$ & - & & \\
\hline 4. Gestión directiva & 2,03 & 0,96 & $0,21^{* *}$ & 0,13 & 0,02 & - & \\
5. Usabilidad & 2,15 & 0,50 & $0,45^{* *}$ & $0,50^{* *}$ & $0,42^{* *}$ & 0,04 & - \\
\hline
\end{tabular}

${ }^{*} p<0,05 ;{ }^{* *} p<0,01$

Fuente: elaboración propia.

\subsection{Modelo de regresión}

Se calcularon dos modelos de regresión. En el primero (M1) se incluyeron las variables relativas al docente (habilidades digitales, usabilidad y utilidad). En el segundo modelo (M2) se agregó la variable relativa al director (gestión directiva). Los resultados mostraron que tanto el modelo $1(F=30,27, p<0,001)$ como el modelo $2(F=26,78, p<0,001)$ explicaron de forma significativa la varianza de los puntajes en las prácticas de enseñanza con apoyo en la tecnología. Se encontró una relación positiva significativa de la habilidad digital, la usabilidad y la gestión directiva con las prácticas de enseñanza. Sin embargo, en el primer modelo la utilidad no aportaba nada de forma significativa a la explicación del constructo (ver la tabla 2). 
Tabla 2. Análisis de regresión para variables del docente y del director que predicen las prácticas de enseñanza con apoyo a la tecnología

\begin{tabular}{lccccc}
\hline & $\boldsymbol{B}$ & $\boldsymbol{E S}$ & $\boldsymbol{B}$ & $\mathbf{R}^{2}$ & $\Delta \mathbf{R}^{2}$ \\
\hline Modelo 1 & & & & 0,30 & \\
Habilidad digital & 0,26 & 0,04 & $0,34^{\star \star *}$ & & \\
Usabilidad & 0,40 & 0,10 & $0,29^{\star * *}$ & & \\
Utilidad & 0,04 & 0,08 & 0,03 & & \\
Modelo 2 & & & & 0,33 & $0,03^{* * *}$ \\
Gestión directiva & 0,14 & 0,04 & $0,19^{* * *}$ & & \\
\hline
\end{tabular}

${ }^{*} p<0,05 ;{ }^{* *} p<0,01 ;{ }^{* * *} p<0,001$

Fuente: elaboración propia.

\section{Conclusiones}

El estudio examina la relación de la habilidad digital, la usabilidad, la utilidad percibida por el docente y la gestión directiva con las prácticas de enseñanza con apoyo de TIC en maestros de educación primaria de escuelas públicas de Sonora (México). Los resultados confirman parcialmente la hipótesis propuesta en el estudio. Adicionalmente, los valores de correlaciones entre las variables muestran tamaños de efectos moderados (utilidad y gestión directiva) o altos (habilidad y usabilidad) entre las variables independientes involucradas en el estudio y las prácticas docentes con apoyo de la tecnología. Estos resultados sugieren implicaciones prácticas a corto y largo plazo de los hallazgos de estudio (Funder y Ozer, 2019).

\subsection{Habilidad digital y prácticas de enseñanza con apoyo de las TIC}

Los resultados del estudio evidencian que la habilidad de los docentes para utilizar las TIC se relaciona positivamente con la frecuencia con que utilizan la tecnología en sus prácticas de enseñanza. Este hallazgo es consistente con lo propuesto en el modelo TPACK (Mishra y Koehler, 2006) y con los resultados empíricos reportados en la literatura (Habibi, Yusop y Razak, 2020; Ifinedo et al., 2020; Kiray et al., 2018). En general este hallazgo reafirma la importancia de conocimientos tecnopedagógicos de los docentes para lograr una integración efectiva de las herramientas tecnológicas en las prácticas de enseñanza (Petko et al., 2018; Siddiq et al., 2016).

\subsection{Usabilidad y prácticas de enseñanza con apoyo de TIC}

En el estudio se encuentra que la usabilidad tiene una influencia positiva en el uso de las TIC como apoyo a la enseñanza por parte de los docentes. Consistente con lo expuesto en la literatura, nuestros hallazgos sugieren que la percepción de la facilidad de uso de las TIC por parte de los docentes favore- 
ce su integración a la enseñanza (Orozco-Cazco et al., 2016). Estos resultados coinciden con lo referido en el modelo TAM, donde se considera la usabilidad una variable crítica para explicar la integración de las TIC por parte de los docentes en sus prácticas de enseñanza (Weng et al., 2018).

\subsection{Utilidad y prácticas de enseñanza con apoyo de TIC}

Contrario a lo esperado en el estudio, la utilidad percibida por los docentes de las TIC no se asocia con el uso de estas herramientas en las prácticas de enseñanza (Mailizar, Almanthari y Maulina, 2021). Estos resultados son diferentes a lo reportado en la literatura (Martín, Sánchez y Costa, 2019). Aunque se necesitan nuevos estudios para aclarar estos resultados, es posible considerar que en la actualidad la mayor parte de los docentes coinciden en pensar que las TIC pueden ser útiles en la enseñanza (Drossel, Eickelman y Gerick, 2017; Sherer et al., 2015), lo cual además es consistente con los resultados descriptivos obtenidos en el estudio.

\subsection{Gestión directiva y prácticas de enseñanza con apoyo de TIC}

Los hallazgos del estudio sugieren que la gestión directiva favorece el uso de TIC en las prácticas de enseñanza por parte de docentes. Este resultado se alinea con lo reportado en la literatura respecto al rol del director en la conformación de las prácticas de enseñanza de los docentes (Arokiasamy et al., 2015; Lim et al., 2015). Se constata que cuando los directores, por medio de su gestión, apoyan el uso de recursos tecnológicos, los docentes los integran en su enseñanza con mayor frecuencia (Ilknur y Yeliz, 2018; Wu et al., 2019).

\subsection{Implicaciones prácticas}

El estudio muestra que tanto variables del docente como de los directores influyen en la integración de las TIC en las prácticas de enseñanza. En particular los resultados sugieren que las habilidades digitales, la percepción de usabilidad de las TIC por parte del docente y la gestión de los directores se relacionan de forma positiva con el uso de las TIC en las prácticas de enseñanza. Desde el punto de vista práctico, los resultados del estudio sugieren varias líneas de acción. En primer lugar, es necesario promover el desarrollo de programas de capacitación de los docentes en competencias tecnopedagógicas relacionadas que faciliten la integración de las TIC en la enseñanza. Esto resulta esencial, dado que en el estudio se muestra que una gran parte de los docentes no ha recibido capacitación sobre el tema en los últimos tres años. En segundo lugar, es imprescindible mejorar la funcionalidad de las TIC en las escuelas para incrementar la percepción de usabilidad. En este sentido, son necesarias acciones de mantenimiento de las tecnologías y organizativas que garanticen el acceso fácil de los maestros a las TIC en las escuelas. Finalmente, es necesario promover una gestión directiva de apoyo al uso de recursos tecnológicos para la enseñanza del profesor. Esto implica que los directores generen condiciones físicas y organizativas en la 
escuela que favorezcan el uso de las TIC por parte de los docentes. Además, involucra que los motiven y los orienten acerca de cómo utilizar las tecnologías para favorecer el aprendizaje de los estudiantes.

\subsection{Limitaciones}

Aunque el estudio contribuye a facilitar la comprensión de los factores vinculados con la integración de las TIC en las prácticas de enseñanza de maestros de educación primaria, presenta limitaciones que provocan que los resultados sean tomados con cautela. En primer lugar, se trata de un diseño transeccional que no permite establecer con claridad relaciones de causa-efecto entre las variables. $\mathrm{Al}$ respecto, se sugieren investigaciones longitudinales o experimentales para profundizar en las relaciones entre las variables involucradas en el estudio. En segundo lugar, los instrumentos utilizados fueron todos de autorreporte. Es importante en próximas investigaciones utilizar otro tipo de instrumentos como de desempeño. Finalmente, aunque la muestra es suficiente para los análisis realizados, es conveniente realizar análisis con muestras más diversas de docentes mexicanos.

\section{Referencias bibliográficas}

Almanasreh, E.; Moles, R. y Chen, T.F. (2019). Evaluation of methods used for estimating content validity. Research in Social and Administrative Pharmacy, 15(2), 214-222. $<$ https://doi.org/10.1016/j.sapharm.2018.03.066>

Almerich, G.; Orellana, N.; Suárez-Rodríguez, J. y Díaz-García, I. (2016). Teachers' information and communication technology competences: A structural approach. Computer \& Education, 100, 110-125. $<$ https://doi.org/10.1016/j.compedu.2016.05.002>

Arokiasamy, A.R.A.; Abdullah, A. e Ismail, A. (2015). Correlation between cultural perceptions, leadership style and ICT usage by school principal in Malaysia. Procedia-Social and Behavioral Science, 176, 319-322. $<$ https://doi.org/10.1016/j.sbspro.2015.01.478>

BAI, B.; WANG, J. y ChAI, C.-S. (2021). Understanding Hong Kong primary school English teachers' continuance intention to teach with ICT. Computer Assisted Language Learning, 34(4), 528-551. <https://doi.org/10.1080/09588221.2019.1627459>

Bas, G.; Kubiatko, M. y Sünbül, A. (2016). Teacher's perceptions towards ICTs in teaching-learning process: Scale validity and reliability study. Computers in Human Behavior, 61, 176-185. <https://doi.org/10.1016/j.chb.2016.03.022>

Beltrán-SÁnchez, J.; García-López, R. y Ramírez-Montoya, M. (2015). Usabilidad y apropiación del programa 'Mi CompuMX' desde la perspectiva de los docentes de primaria. Ponencia presentada en el XIII Congreso Nacional de Investigación, México, COMIE.

Burns, T. y GotTsChaLk, F. (2019). Educating 21st century children: Emotional wellbeing in the digital age. OECD. 
Byrne, B. (2016). Structural Equation Modeling with AMOS: Basic concepts, applications, and programming. 2a ed. Nueva York: Routledge.

Cataldo, A. (2015). Limitaciones y oportunidades del Modelo de Aceptación Tecnológica (TAM). Copiapó: Universidad de Atacama.

Chai, C.; Koh, J.; Natarajan, U.; Tsai, P.; Ramli, M. y Widodo, A. (2017). Surveying Indonesian teachers' design belief and TPACK for 21st Century Oriented Learning. En W. Chen; J.-C. YAng; A.F. Mohd Ayub; S.L. Wong y A. Mitrovic (eds.). Proceedings of the 25th International Conference on Computers in Education (pp. 1001-1006). Recuperado de <https://repository.nie.edu.sg/bitstream/ 10497/19433/1/ICCE-2017-1001.pdf>.

Comi, S.; Argentin, G.; Gui, M.; Origo, F. y Pagani, L. (2017). Is it the way they use it?: Teachers, ICT and student achievement. Economics of Education Review, 56, 24-39. <https://doi.org/10.1016/j.econedurev.2016.11.007>

Daly, A.J.; Liou, Y.-H. y MoolenaAr, N.M. (2014). The principal connection: Trust and innovative climate in a network of reform. En D. Van Maele; P. Forsyth y M. Van Houtte (eds.). Trust and School life (pp. 285-311). Dordrecht: Springer. <https://doi.org/10.1007/978-94-017-8014-8_13>

Davis, F. (1989). Perceived usefulness, perceived ease of use, and user acceptance of information technology. MIS Quarterly, 13(3), 319-340. $<$ https://doi.org/10.2307/249008>

Drossel, K.; Eickelman, B. y Gerick, J. (2017). Predictors of teachers' use of ICT in school: The relevance of school characteristics, teachers' attitudes and teacher collaboration. Education and Information Technologies, 22, 551-573. <https://doi.org/10.1007/s10639-016-9476-y>

Funder, D.C. y Ozer, D.J. (2019). Evaluating effect size in psychological research: Sense and nonsense. Advances in Methods and Practices in Psychological Science, 2(2), 156-168. <https://doi.org/10.1177/2515245919847202>

Gil-Flores, J.; Rodríguez-SAntero, J. y Torres-Gordillo, J.-J. (2017). Factors that explain the use of ICT in secondary-education classrooms: The role of teacher characteristics and school infrastructure. Computer in Human Behavior, 68, 441-449. <https://doi.org/10.1016/j.chb.2016.11.057>

Glasserman-Morales, L. y Manzano-Torres, J. (2016). Diagnóstico de las habilidades digitales y prácticas pedagógicas de los docentes en educación primaria en el marco del programa Mi CompuMx. Apertura, 8(1), 1-17. Recuperado de <http://www.udg virtual.udg.mx/apertura/index.php/apertura/article/view/820>.

Gómez-Domínguez, C.; Ramírez-Romero, J.; Martínez-González, O. y ChucPIÑA, I. (2019). Uso de las TIC en la enseñanza del inglés en las primarias públicas. Revista de Estudios y Experiencias en Educación, 18(36), 75-94. <http://dx.doi.org/10.21703/rexe.20191836gomez4>

Habibi, A.; Yusop, F.D. y RazaK, R.A. (2020). The role of TPACK in affecting preservice language teachers' ICT integration during teaching practices: Indonesian context. Education and Information Technology, 25, 1929-1949. $<$ https://doi.org/10.1007/s10639-019-10040-2>

Hernández-Martín, A. y Martín de Arriba, J. (2017). Concepciones de los docentes no universitarios sobre el aprendizaje colaborativo con TIC. Educación XX1, 20(1), 185-208.

<https://doi.org/10.5944/educxx1.17508> 
Hu, X.; Gong, Y.; LaI, C. y Leung, F.K.S. (2018). The relationship between ICT and student literacy in mathematics, reading, and science across 44 countries: A multilevel analysis. Computer \& Education, 125, 1-13. $<$ https://doi.org/10.1016/j.compedu.2018.05.021>

IFINEDO, E.; RikalA, J. y HäMÄLÄINEN, T. (2020). Factors affecting Nigerian teacher educators' technology integration: Considering characteristics, knowledge constructs, ICT practices and beliefs. Computer \& Education, 146, 1-17. $<$ https://doi.org/10.10 16/j.compedu.2019.103760>

IlknuR, M. y Yeliz, K. (2018). School principals' and teachers' views on teacher performance evaluation. International Journal of Progressive Education, 14(5), 77-88. <https://doi.org/10.29329/ijpe.2018.157.7>

Instituto Nacional de Estadística y Geografía (2018). Anuario estadístico y geográfico de los Estados Unidos Mexicanos 2017. Aguascalientes: Instituto Nacional de Estadística y Geografía.

Johnson, R.B. y CHRISTENSEn, L. (2017). Educational research: Quantitative, qualitative and mixed approaches. 6a ed. Thousand Oaks, CA: SAGE Publications.

Kars, M. e InANDI, Y. (2018). Relationship between school principals' leadership behaviors and teachers' organizational trust. Eurasian Journal of Educational Research, 74, 145-164.

Kiray, S.; Celik, I. y Colakoglu, M. (2018). TPACK self-efficacy perceptions of science teachers: A structural equation modeling study. Education and Science, 43(195), 253-268. <https://doi.org/10.15390/EB.2018.7538>

LAWRENCE, J.E. y TAR, U.A. (2018). Factors that influence teachers' adoption and integration of ICT in teaching/learning process. Educational Media International, 55(1), 79-195. <https://doi.org/10.1080/09523987.2018.1439712>

Lim, N.; Gronlund, A. y Andersson, A. (2015). Cloud computing: The beliefs and perceptions of Swedish school principals. Computers \& Education, 84, 90-100. <https://doi.org/10.1016/j.compedu.2015.01.009>

Machado, L. y Chung, C. (2015). Integrating Technology: The Principals' Role and Effect. International Education Studies, 8(5), 43-55. <http://dx.doi.org/10.5539/ies.v8n5p43>

MadDox, T. (2004). Analysis of technology competence of K-12 Teachers in a selected school district in Mississippi. Dissertation Archive, 1845. Universidad de Mississippi.

Mailizar, M.; Almanthari, A. y Maulina, S. (2021). Examining teachers' behavioral intention to use e-learning in teaching of mathematics: An extended TAM model. Contemporary Educational Technology, 13(2), 1-16. $<$ https://doi.org/10.30935/cedtech/9709>

Martín García, A.; Sánchez Gómez, M.C. y Costa, A.P. (2019). Percepción de Blended Learning en profesores universitarios de distintos ámbitos disciplinares. Revista Lusófona de Educação, 44(44), 117-133. <https://doi.org/10.24140/issn.1645-7250.rle44.07>

Mendoza-Orellana, A. (2019). El rol del director en la mejora y la eficacia de las escuelas de Iberoamérica: Una revisión de la literatura. Pontificia Universidad Católica del Perú [tesis de maestría]. Recuperado de <http://hdl.handle.net/20.500. 12404/14261>. 
Mishra, P. y Koehler, M.J. (2006). Technological pedagogical content knowledge: A framework for integrating technology in teacher knowledge. Teachers College Record, 108(6), 1017-1054.

Muli, A.; Musyoki, D. y Muema, J. (2017). The influence of principals' related factors on ICT integration in the management of public secondary schools in Kitui County, Kenya. Journal of Educational Policy and Entrepreneurial Research, 4(1), 13-24.

Navaridas-Nalda, F; Emeterio, M.C.-S.; Fernández-Ortiz, R. y Arias-Oliva, M. (2020). The strategic influence of school principal leadership in the digital transformation of schools. Computer in Human Behavior, 112, artículo e106481. <https://doi.org/10.1016/j.chb.2020.106481>

Odu, E.O. y Mireku, A.A. (2016). The influence of Information and Communication Technology (ICT) in improving teaching of environmental education. Journal of Human Ecology, 55(1-2), 1-8. <https://doi.org/10.1080/09709274.2016.11907003>

Organization for Economic Co-operation and Development (2017). PISA 2015 Results (Volume III): Students' Well-Being. París: OECD Publishing. <https://doi.org/10.1787/9789264273856-en>

Organización de las Naciones Unidas para la Educación, la Ciencia y la Cultura (2014). El liderazgo escolar en América Latina y el Caribe: Un estado del arte con base a ocho sistemas escolares de la región. Santiago: OREALC/UNESCO. Recuperado de <https://unesdoc.unesco.org/ark:/48223/pf0000232799>.

Orozco-Cazco, G.; Cabezas-González, M.; Martínez-Abad, F.; DelgadoAltamirano, J. y Solís-Mazon, M. (2016). Determining factors in acceptance of ICT by the university faculty in their teaching practice. Proceedings of the Fourth International Conference on Technological Ecosystems for Enhancing Multiculturality.

Petko, D.; Prasse, D. y Cantieni, A. (2018). The interplay of school readiness and teacher readiness for educational technology integration: A structural equation model. Computers in the Schools, 35(1), 1-18. <https://doi.org/10.1080/073 80569.2018.1428007>

Ríos-Ariza, J.; Gómez-Barajas, E. y Rojas-Polanco, M. (2018). Valoración de competencias TIC del profesorado universitario: Un caso en Chile. Pixel-Bit, 52, 55-65. <http://dx.doi.org/10.12795/pixelbit.2018.i52.04>

Sánchez-Prieto, J.; Olmos-Migueláñez, S. y García-Peñalvo, F. (2015). Intención de uso de tecnologías móviles entre los profesores en formación: Aplicación de un modelo de adopción tecnológica basado en TAM con los constructos compatibilidad y resistencia al cambio. En M.R. Rodrigues; M.L. Nistal y M. FIgueIRedo (eds.). Atas do XVII Simpósio Internacional de Informática Educativa (pp. 260-267). Escola Superior de Educação do Instituto Politécnico de Setúbal. Recuperado de <https://gredos.usal.es/handle/10366/126981>.

Secretaría de Educación Pública (2017). Perfil, parámetros e indicadores para personal con funciones de dirección y de supervisión en educación básica. Recuperado de $<$ https://es.slideshare.net/ma072001/perfil-parmetros-e-indicadores-para-elpersonal-con-funciones-de-direccin-y-de-supervisin-en-la-educacin-bsica>.

Sherer, R.; Siddie, F. y Teo, T. (2015). Becoming more specific: Measuring and modeling Teacher's perceived usefulness of ICT in the context of teaching and learning. Computer \& Education, 88, 202-214. <https://doi.org/10.1016/j.compedu.2015.05.005> 
Siddie, F.; Sherer, R. y Tondeur, J. (2016). Teachers' emphasis on developing students' digital information and communication skills (TEDDICS): A new construct in 21st century education. Computers \& Education, 92(93), 1-14. $<$ https://doi.org/10.1016/ j.compedu.2015.10.006>

Skryabin, M.; Zhang, J.; Liv, L. y Zhang, D. (2015). How the ICT development level and usage influence student achievement on reading, mathematics, and science. Computer \& Education, 85, 49-58. $<$ https://doi.org/10.1016/j.compedu.2015.02.004>

Stein, K.C.; Macaluso, M. y Stanulis, R.N. (2016). The interplay between principal leadership and teacher leader efficacy. Journal of School Leadership, 26(6), 1002-1032. <https://doi.org/10.1177/105268461602600605>

Tarhini, A.; Elyas, T.; Ali, M. y Al-Salti, Z. (2016). Technology, demographic, characteristics, and E-learning acceptance: A conceptual model based on extended Technology Acceptance Model. Higher Education Studies, 6(3), 72-89. $<$ https://doi.org/10.5539/hes.v6n3p72>

Weng, F.; YANG, R.; Ho, H. y Su, H. (2018). A TAM-based study of the attitude towards use intention of multimedia among school teachers. Applied System Innovation, 1(36), 1-9. $<$ https://doi.org/10.3390/asi1030036>

Wu, B.; Yu, Y. y Hu, Y. (2019). How does principal e-leadership affect ICT transformation across different stages in K-12 education: Perspectives from teachers in Shanghai. British Journal of Educational Technology, 50(3), 1210-1225. $<$ https://doi.org/10.1111/bjet.12766>

XIAO, Y.; Lin, Y. y Hu, J. (2019). Regression analysis of ICT impact factors on early adolescents' reading proficiency in five high-performing countries. Frontiers in Psychology, 10, artículo e1646.

$<$ https://doi.org/10.3389/fpsyg.2019.01646> 